\title{
On statistical convergence in quasi-metric spaces
}

https://doi.org/10.1515/dema-2019-0019

Received January 21, 2019; accepted May 7, 2019

\begin{abstract}
A quasi-metric is a distance function which satisfies the triangle inequality but is not symmetric in general. Quasi-metrics are a subject of comprehensive investigation both in pure and applied mathematics in areas such as in functional analysis, topology and computer science. The main purpose of this paper is to extend the convergence and Cauchy conditions in a quasi-metric space by using the notion of asymptotic density. Furthermore, some results obtained are related to completeness, compactness and precompactness in this setting using statistically Cauchy sequences.
\end{abstract}

Keywords: quasi-metric space, asymptotic density, statistical convergence, forward and backward Cauchy sequences

MSC: P54A05, 54E50, 40G15

\section{Introduction}

Let $X$ be a set. A non-negative real-valued function $\rho$ on $X^{2}$ is called as a quasi-metric on $X$ if it satisfies the following axioms:

1. $\rho(x, y)=\rho(y, x)=0$ if and only if $x=y$,

2. $\rho(x, y) \leq \rho(x, z)+\rho(z, y)$,

for all $x, y, z \in X$. That is, it is possible $\rho(x, y) \neq \rho(y, x)$ for some $x, y \in X$. In this case, $(X, \rho)$, or simply $X$, is called a quasi-metric space (also known as an asymmetric metric space). Firstly, the term quasi-metric was proposed by Wilson [1]. This structure was investigated by many authors using different names (see, for example, [2]). Later, quasi-metrics became a subject of intensive research in the context of functional analysis, topology and theoretical computer science.

Every quasi-metric has a conjugate quasi-metric. The function $\bar{\rho}$ defined by $\bar{\rho}(x, y)=\rho(y, x)$ for all $x, y \in$ $X$ is also a quasi-metric on $X$ and is called the conjugate quasi-metric of $\rho$. Also, the mapping $\rho^{s}(x, y)=$ $\max \{\rho(x, y), \bar{\rho}(x, y)\}$ is a metric on $X$. The inequalities $\rho(x, y) \leq \rho^{s}(x, y)$ and $\bar{\rho}(x, y) \leq \rho^{s}(x, y)$ hold for all $x, y \in X$.

This modification in the definition of a metric space changes the whole theory, especially related to completeness, compactness and precompactness. The other primary difference with the metric case is that in a quasi-metric space some concepts such as convergence have two distinct forms. We call the structures obtained using the original quasi-metric as the left structures and the structures obtained using the conjugate quasi-metric as the right structures.

\footnotetext{
${ }^{\star}$ Corresponding Author: Merve İlkhan: Düzce University, Faculty of Arts and Sciences, Department of Mathematics, 81620, Düzce, Turkey; E-mail: merveilkhan@gmail.com

Emrah Evren Kara: Düzce University, Faculty of Arts and Sciences, Department of Mathematics, 81620, Düzce, Turkey;

E-mail: karaeevren@gmail.com
} 
There are two natural topologies on a quasi-metric space $(X, \rho)$. Each quasi-metric $\rho$ naturally induces a topology $\tau_{\rho}$ whose base consists of all left $\rho$-open balls

$$
B_{\rho}(x, \varepsilon)=\{y \in X: \rho(x, y)<\varepsilon\}, \varepsilon>0, x \in X .
$$

The topology $\tau_{\bar{\rho}}$ induced by the conjugate quasi-metric is defined in a similar way- its base consists of all right $\rho$-open balls

$$
B_{\bar{\rho}}(x, \varepsilon)=\{y \in X: \bar{\rho}(x, y)<\varepsilon\}, \varepsilon>0, x \in X .
$$

Every quasi-metric space $X$ can be viewed as a bitopological space (see [3]) with respect to the topologies $\tau_{\rho}$ and $\tau_{\bar{\rho}}$. It is clear that the topologies $\tau_{\rho}$ and $\tau_{\bar{\rho}}$ satisfy the $T_{0}$ separation axiom. These topologies are $T_{1}$ if and only if $\rho(x, y)>0$ whenever $x \neq y$.

The convergence of a sequence $\left(x_{n}\right)$ in a quasi-metric space $X$ with respect to $\tau_{\rho}\left(\tau_{\bar{\rho}}\right)$ is called left (right) $\rho$-convergence which means

$$
x_{n} \stackrel{1-\rho}{\longrightarrow} x \Leftrightarrow \rho\left(x, x_{n}\right) \rightarrow 0 \quad\left(x_{n} \stackrel{\mathrm{r}-\rho}{\longrightarrow} x \Leftrightarrow \rho\left(x_{n}, x\right) \rightarrow 0\right) .
$$

Throughout the paper, $\mathbb{N}$ and $\mathbb{R}$ denote the set of all natural and real numbers, respectively.

Kelly [3] was the first to give a definition of a Cauchy sequence in a quasi-metric space and the corresponding completeness as given in the items (1) below. He observed that this definition has the disadvantage that a convergent sequence need not be Cauchy. Later, the authors of [4] introduced seven different notions of a Cauchy sequence in a quasi-metric space from which we mention the following three:

A sequence $\left(x_{n}\right)$ in $(X, \rho)$ is said to be:

1. left (right) $K$-Cauchy if for every $\varepsilon>0$ there exists a natural number $n_{0} \in \mathbb{N}$ such that $\rho\left(x_{m}, x_{n}\right)<\varepsilon$ $\left(\rho\left(x_{n}, x_{m}\right)<\varepsilon\right)$ for $n \geq m \geq n_{0}$;

2. weakly left (right) $K$-Cauchy if for every $\varepsilon>0$ there exists a natural number $n_{0} \in \mathbb{N}$ such that $\rho\left(x_{n_{0}}, x_{n}\right)<\varepsilon$ $\left(\rho\left(x_{n}, x_{n_{0}}\right)<\varepsilon\right)$ for $n \geq n_{0}$;

3. left (right) $\rho$-Cauchy if for every $\varepsilon>0$ there exist a point $x \in X$ and a natural number $n_{0} \in \mathbb{N}$ such that $\rho\left(x, x_{n}\right)<\varepsilon\left(\rho\left(x_{n}, x\right)<\varepsilon\right)$ for $n \geq n_{0}$.

In the metric case each of the above definitions yields the usual notion of a Cauchy sequence. Also notice that only that given in (3) has the property that a convergent sequence in a quasi-metric space is Cauchy (in fact this is true for all seven notions of a Cauchy sequence given in [4]).

In the case of a quasi-metric space, there are several completeness notions by asking that each corresponding Cauchy sequence converges with respect to the topologies $\tau_{\rho}$ or $\tau_{\bar{\rho}}$.

A quasi-metric space $(X, \rho)$ is said to be:

1. left $K$-sequentially complete if every left $K$-Cauchy sequence in $X$ is left $\rho$-convergent;

2. weakly left $K$-sequentially complete if every weakly left $K$-Cauchy sequence in $X$ is left $\rho$-convergent;

3. left $\rho$-sequentially complete if every left $\rho$-Cauchy sequence in $X$ is left $\rho$-convergent.

A set $Y$ in a quasi-metric space $(X, \rho)$ is said to be precompact if for every $\varepsilon>0$, there exists a finite subset $F$ of $Y$ such that

$$
Y \subset \bigcup\left\{B_{\rho}(x, \varepsilon): x \in F\right\} .
$$

If in this definition we further suppose that $F$ is a finite subset of $X$, then $Y$ is called outside precompact. Obviously, a precompact set is outside precompact, but the converse is not necessarily true in arbitrary quasimetric spaces. In metric spaces both notions agree. Also, a quasi-metric space $(X, \rho)$ is called hereditarily precompact if every subset of $X$ is precompact.

For the definitions and results related to quasi-metric spaces, one can consult [4-11] and the references therein.

The idea of statistical convergence which is an extension of usual convergence goes back to Zygmund [12]. The statistical convergence for a real-valued sequence was introduced by Fast [13]. The formal definition is based on the asymptotic density of a subset $A$ in $\mathbb{N}$ which is defined as $\delta(A)=\lim _{n \rightarrow \infty} \frac{1}{n}|\{k \leq n: k \in A\}|$ if this limit exists. Here and throughout this paper, $|B|$ denotes the number of elements in $B$. Note that $0 \leq \delta(A) \leq 1$ 
and $\delta(\mathbb{N} \backslash A)=1-\delta(A)$ for all $A \subset \mathbb{N}$. In [14], the author studied the concept as a summability method. Later, this concept was investigated and linked with the summability theory in many papers (see, for example, [1526]).

In this study, we give some basic definitions and obtain some fundamental results related to statistical convergence on quasi-metric spaces. By defining two new types of statistically Cauchy sequences (called weakly left (right) $K$-statistical Cauchy and left (right) $\rho$-statistical Cauchy), the relations between these sequences are explained with examples. In contrast to the metric spaces case, it is observed that there are some differences related to statistical concepts. For example, the statistical limit of a sequence in a quasi-metric space is not unique. Furthermore, some interesting results are obtained related to completeness (in some sense), compactness and precompactness in this setting by using these statistically Cauchy sequences. In the last part of the paper, some summability type theorems are presented.

\section{Statistical convergence in quasi-metric spaces}

It is defined in [27] that a sequence $\left(x_{n}\right)$ in a quasi-metric space $(X, \rho)$ is forward (backward) statistically convergent to $x \in X$, if for every $\varepsilon>0$, we have $\delta\left(\left\{n: \rho\left(x, x_{n}\right) \geq \varepsilon\right\}\right)=0\left(\delta\left(\left\{n: \rho\left(x_{n}, x\right) \geq \varepsilon\right\}\right)=0\right)$. We call this sequence a left (right) $\rho$-statistically convergent sequence and the point $x$ a left (right) $\rho$-statistical limit of $\left(x_{n}\right)$.

Remark 2.1. If a sequence $\left(x_{n}\right)$ in a quasi-metric space $(X, \rho)$ is statistically convergent to a point $x \in X$ with respect to the metric $\rho^{s}$, then it is both left and right $\rho$-statistically convergent to $x$ which follows from the inclusions $\left\{n: \rho^{s}\left(x, x_{n}\right)<\varepsilon\right\} \subseteq\left\{n: \rho\left(x, x_{n}\right)<\varepsilon\right\}$ and $\left\{n: \rho^{s}\left(x, x_{n}\right)<\varepsilon\right\} \subseteq\left\{n: \rho\left(x_{n}, x\right)<\varepsilon\right\}$ for any $\varepsilon>0$. But, the converse is not true.

Example 2.2. Let $X=\mathbb{R}$ and $\rho$ be the quasi-metric on $X$ defined by

$$
\rho(x, y)=\left\{\begin{array}{cc}
y-x, & \text { if } x<y, \\
0, & \text { if } x \geq y .
\end{array}\right.
$$

Consider the sequence $\left(x_{n}\right)=\left((-1)^{n}\right)$. Then, we have $\rho\left(1, x_{n}\right)=0$ and $\rho\left(x_{n},-1\right)=0$ for all $n \in \mathbb{N}$. It follows that given any $\varepsilon>0, \delta\left(\left\{n: \rho\left(1, x_{n}\right)<\varepsilon\right\}\right)=1$ and $\delta\left(\left\{n: \rho\left(x_{n},-1\right)<\varepsilon\right\}\right)=1$ which imply $\left(x_{n}\right)$ is left $\rho$-statistically convergent to 1 and right $\rho$-statistically convergent to -1 , respectively. But, since $\rho^{s}$ is the absolute value metric on $\mathbb{R}$, we have

$$
\delta\left(\left\{n: \rho^{s}\left(x, x_{n}\right)<1\right\}\right)=\left\{\begin{array}{cc}
\frac{1}{2}, & \text { if }|x|<2 \text { and } x \neq 0, \\
0, & \text { otherwise, }
\end{array}\right.
$$

which means this sequence is not statistically convergent with respect to $\rho^{s}$.

Remark 2.3. The left (right) $\rho$-statistical limit of a sequence $\left(x_{n}\right)$ in a quasi-metric space $(X, \rho)$ is not unique in general. This can be seen from the preceding example using the fact that, for all $n \in \mathbb{N}$ and $x \in[1, \infty)$ $(x \in(-\infty,-1])$, we have $\rho\left(x, x_{n}\right)=0\left(\rho\left(x_{n}, x\right)=0\right)$.

Remark 2.4. There exist sequences in a quasi-metric space which are left $\rho$-statistically convergent but not right $\rho$-statistically convergent.

Example 2.5. Let $(X, \rho)$ be the quasi-metric space in Example 2.2. The sequence $\left(x_{n}\right)=(-n)$ is left $\rho$-statistically convergent to every point in $X$ but not right $\rho$-statistically convergent to any point in $X$.

The authors of the paper [28] extended the notion of a forward (backward) Cauchy sequence (also known as a left (right) $K$-Cauchy sequence) by using an ideal of $\mathbb{N}$. In this definition, if we choose the ideal consisting of subsets of $\mathbb{N}$ with asymptotic density zero (in this case the ideal convergence coincides with statistical 
convergence), then we obtain the following condition:

For every $\varepsilon>0$ there exists a subset $M \subseteq \mathbb{N}$ with $\delta(M)=1$ such that the equality $\delta\left(\left\{n: \rho\left(x_{k}, x_{n}\right) \geq \varepsilon\right\}\right)=0$ $\left(\delta\left(\left\{n: \rho\left(x_{n}, x_{k}\right) \geq \varepsilon\right\}\right)=0\right)$ holds for every $k \in M$.

We call a sequence satisfying this condition a left (right) $K$-statistically Cauchy sequence.

Lemma 2.6. If a sequence $\left(x_{n}\right)$ in a quasi-metric space $(X, \rho)$ is a left (right) $K$-Cauchy sequence, then it is a left (right) K-statistically Cauchy sequence.

Proof. Let $\left(x_{n}\right)$ be a left $K$-Cauchy sequence in $X$. Then, given any $\varepsilon>0$ there exits $n_{0} \in \mathbb{N}$ such that $\rho\left(x_{m}, x_{n}\right)<$ $\varepsilon$ for every $n \geq m \geq n_{0}$. Put $M=\left\{n_{0}, n_{0}+1, \ldots\right\}$. It is clear that $\delta(M)=1$. Choose $k \in M$. The inclusion

$$
\left\{n>k: \rho\left(x_{k}, x_{n}\right)<\varepsilon\right\} \subset\left\{n: \rho\left(x_{k}, x_{n}\right)<\varepsilon\right\}
$$

implies that $\left(x_{n}\right)$ is a left $K$-statistically Cauchy sequence since the asymptotic density of the set in the left side is 1.

The following example shows that the converse is not true in general.

Example 2.7. Let $X=\mathbb{R}$ and $\rho$ be the quasi-metric on $X$ defined by

$$
\rho(x, y)=\left\{\begin{array}{cc}
x-y, & \text { if } x \geq y, \\
1, & \text { if } x<y .
\end{array}\right.
$$

Consider the sequence $\left(x_{n}\right)$ given as

$$
x_{n}=\left\{\begin{array}{cc}
1, & \text { if } n=k^{2},(k \in \mathbb{N}), \\
\frac{1}{n}, & \text { if } n \neq k^{2} .
\end{array}\right.
$$

Choose $n, m \in \mathbb{N}$ such that $n>m$ with $n=p^{2}$ for some $p \in \mathbb{N}$ and $m \neq r^{2}$ for every $r \in \mathbb{N}$. Then, we obtain $\rho\left(x_{m}, x_{n}\right)=1$ which implies that $\left(x_{n}\right)$ is not a left $K$-Cauchy sequence. On the other hand, given any $\varepsilon>0$ we can find a natural number $n_{0}$ such that $\frac{1}{n_{0}}<\varepsilon$ and $n_{0} \neq r^{2}$ for every $r \in \mathbb{N}$. Hence, we obtain $\rho\left(x_{m}, x_{n}\right)=\frac{1}{m}-\frac{1}{n}<\varepsilon$ for $n \geq m \geq n_{0}$. This proves that the subsequence $\left(\frac{1}{n}\right)_{n \in A_{0}}$ of $\left(x_{n}\right)$ is a left $K$-Cauchy sequence, where $A_{0}=\left\{n: n \neq k^{2}, k \in \mathbb{N}\right\}$. Thus, $\left(x_{n}\right)$ is a left $K$-statistically Cauchy sequence.

Definition 2.8. A sequence $\left(x_{n}\right)$ in a quasi-metric space $(X, \rho)$ is said to be:

1. weakly left (right) $K$-statistical Cauchy if for every $\varepsilon>0$ there exits $n_{0} \in \mathbb{N}$ such that $\delta\left(\left\{n: \rho\left(x_{n_{0}}, x_{n}\right) \geq\right.\right.$ $\varepsilon\})=0\left(\delta\left(\left\{n: \rho\left(x_{n}, x_{n_{0}}\right) \geq \varepsilon\right\}\right)=0\right)$;

2. left (right) $\rho$-statistical Cauchy if for every $\varepsilon>0$ there exits $x \in X$ such that $\delta\left(\left\{n: \rho\left(x, x_{n}\right) \geq \varepsilon\right\}\right)=0$ $\left(\delta\left(\left\{n: \rho\left(x_{n}, x\right) \geq \varepsilon\right\}\right)=0\right)$.

We state our results for the left structures, however, similar results can be obtained for right structures.

Theorem 2.9. A sequence $\left(x_{n}\right)$ in a quasi-metric space $(X, \rho)$ is left $\rho$-statistically convergent (left $K$-statistical Cauchy, weakly left $K$-statistical Cauchy, left $\rho$-statistical Cauchy) if and only if there exists a subset $K=\left\{n_{1}<\right.$ $\left.n_{2}<\ldots<n_{k} \ldots\right\}$ of $\mathbb{N}$ such that $\delta(K)=1$ and the subsequence $\left(x_{n_{k}}\right)$ is left $\rho$-convergent (left $K$-Cauchy, weakly left $K$-Cauchy, left $\rho$-Cauchy).

Proof. We prove the theorem for a left $\rho$-statistically convergent sequence. The other statements can be proved in a similar way.

Suppose that $\left(x_{n}\right)$ is left $\rho$-statistically convergent to a point $x$ in $X$. Then, we have $\delta\left(A_{l}\right)=1$, where $A_{l}=\left\{n: \rho\left(x, x_{n}\right)<\frac{1}{l}\right\}$ for all $l \in \mathbb{N}$. We can construct an increasing sequence of natural numbers $i_{1}<i_{2}<$ $\ldots<i_{l}<\ldots$ such that $i_{l} \in A_{l}$ and

$$
1-\frac{1}{l}<\frac{1}{n}\left|\left\{k \leq n: \rho\left(x, x_{k}\right)<\frac{1}{l}\right\}\right|
$$


for every $n \geq i_{l}(l \in \mathbb{N})$. Put

$$
A=\left\{n: 1 \leq n<i_{1}\right\} \cup\left[\bigcup_{l \in \mathbb{N}}\left\{n: i_{l} \leq n<i_{l+1}\right\} \cap A_{l}\right] .
$$

By (1), for all $l \in \mathbb{N}$ and $i_{l} \leq n<i_{l+1}$ the inclusion

$$
\left\{k \leq n: \rho\left(x, x_{k}\right)<\frac{1}{l}\right\} \subset\{k \leq n: k \in A\}
$$

implies

$$
1-\frac{1}{l}<\frac{1}{n}\left|\left\{k \leq n: \rho\left(x, x_{k}\right)<\frac{1}{l}\right\}\right| \leq \frac{1}{n}|\{k \leq n: k \in A\}|,
$$

which means $\delta(A)=1$. Given any $\varepsilon>0$, there exists an $l \in \mathbb{N}$ such that $\frac{1}{l}<\varepsilon$. Choose $n \in A$ with $n \geq i_{l}$. Then, we can find a natural number $r$ with $r \geq l$ such that $i_{r} \leq n<i_{r+1}$ and $n \in A_{r}$. It follows that $\rho\left(x, x_{n}\right)<\varepsilon$. This proves our assertion.

Conversely, let $\delta\left(\left\{n_{k}: k \in \mathbb{N}\right\}\right)=1$ and let the subsequence $\left(x_{n_{k}}\right)$ be left $\rho$-convergent to a point $x \in X$. Then, for $\varepsilon>0$, there exists a $k_{0} \in \mathbb{N}$ such that $\rho\left(x, x_{n_{k}}\right)<\varepsilon$ for every $k \geq k_{0}$. It is clear that the inclusion

$$
\left\{n: \rho\left(x, x_{n}\right) \geq \varepsilon\right\} \subset \mathbb{N}-\left\{n_{k_{0}}, n_{k_{0}+1}, \ldots\right\}
$$

holds. Then, we obtain that $\delta\left(\left\{n: \rho\left(x, x_{n}\right) \geq \varepsilon\right\}\right)=0$ since the natural density of the set $\mathbb{N}-\left\{n_{k_{0}}, n_{k_{0}+1}, \ldots\right\}$ is zero. Consequently, the sequence $\left(x_{n}\right)$ is left $\rho$-statistically convergent to $x$.

Corollary 2.10. If a sequence in a quasi-metric space $(X, \rho)$ is left $\rho$-statistically convergent (left $K$-statistical Cauchy, weakly left $K$-statistical Cauchy, left $\rho$-statistical Cauchy), then it has a left $\rho$-convergent (left $K$-Cauchy, weakly left $K$-Cauchy, left $\rho$-Cauchy) subsequence.

It is obvious from definitions that the left $\rho$-convergence of a sequence implies that it is left $\rho$-statistically convergent to the same point. Also, if a sequence is left $\rho$-Cauchy or weakly left $K$-Cauchy, then it is a left $\rho$ statistically Cauchy sequence or a weakly left $K$-statistically Cauchy sequence, respectively. But the converse statements are false. This is shown by Example 2.26. Furthermore, a left $\rho$-statistically convergent sequence and a weakly left $K$-statistically Cauchy sequence is left $\rho$-statistical Cauchy. However, a left $\rho$-statistically Cauchy sequence is not a left $\rho$-statistically convergent sequence or a weakly left $K$-statistically Cauchy sequence in general (see Example 2.11 and Example 2.12).

Example 2.11. Let $X=[0,1]$ and $\rho$ be the quasi-metric on $X$ defined by

$$
\rho(x, y)= \begin{cases}0, & \text { if } x \leq y \\ 1, & \text { if } x>y\end{cases}
$$

Consider the sequence $\left(x_{n}\right)$ given as

$$
x_{n}= \begin{cases}\frac{1}{2}+\frac{1}{2^{n}}, & \text { if } n \text { is odd }, \\ \frac{1}{3}+\frac{1}{3^{n}}, & \text { if } n \text { is even } .\end{cases}
$$

This sequence is left $\rho$-convergent (see Example 1 in [4]). Consequently, it is left $\rho$-statistically convergent and hence it is a left $\rho$-statistically Cauchy sequence in X. However, $\left(x_{n}\right)$ is not a weakly left $K$-statistically Cauchy sequence in $X$. To observe that take any $N \in \mathbb{N}$. If $N$ is odd, then we have

$$
\left\{k \leq n: \rho\left(x_{N}, x_{k}\right)<\frac{1}{2}\right\} \subseteq\{k \leq N: k \text { is odd }\} .
$$

Hence, the inequality

$$
\left|\left\{k \leq n: \rho\left(x_{N}, x_{k}\right)<\frac{1}{2}\right\}\right| \leq \frac{N+1}{2}
$$


holds. Accordingly, we have $\lim _{n \rightarrow \infty} \frac{1}{n}\left|\left\{k \leq n: \rho\left(x_{N}, x_{k}\right)<\frac{1}{2}\right\}\right|=0$. In the case that $N$ is an even number, we obtain the inclusions

$$
\left\{k \leq n: \rho\left(x_{N}, x_{k}\right)<\frac{1}{2}\right\} \subseteq\{k \leq n: k \text { is odd }\} \cup\{k \leq N: k \text { is even }\}
$$

which implies

$$
\left|\left\{k \leq n: \rho\left(x_{N}, x_{k}\right)<\frac{1}{2}\right\}\right| \leq \mid\{k \leq n: k \text { is odd }\} \mid+\frac{N}{2} .
$$

We conclude that $\lim _{n \rightarrow \infty} \frac{1}{n}\left|\left\{k \leq n: \rho\left(x_{N}, x_{k}\right)<\frac{1}{2}\right\}\right| \leq \frac{1}{2}$. This shows that $\left(x_{n}\right)$ is not a weakly left $K$-statistically Cauchy sequence.

Example 2.12. Let $X=\{0\} \cup\left\{\frac{1}{n}: n \in \mathbb{N}\right\}$ and $\rho$ be a quasi-metric on $X$ such that

$$
\rho(x, y)=\left\{\begin{array}{cc}
1, & \text { if } n \text { is even } y \neq 1 / n \text { and } x=1 / n, \\
1 / n, & \text { if } n \text { is odd } y \neq 1 / n \text { and } x=1 / n, \\
1 / n, & \text { if } n \text { is even } y=1 / n \text { and } x=0, \\
1, & \text { if } n \text { is odd } y=1 / n \text { and } x=0, \\
0, & \text { if } x=y .
\end{array}\right.
$$

Consider the sequence $\left(\frac{1}{n}\right)$ in X. Given any $\varepsilon>0$, there exits an odd number $n_{0} \in \mathbb{N}$ such that $\frac{1}{n_{0}}<\varepsilon$ and $\left|\left\{k \leq n: \rho\left(\frac{1}{n_{0}}, \frac{1}{n}\right)<\varepsilon\right\}\right|=n$. Hence, we have $\lim _{n \rightarrow \infty} \frac{1}{n}\left|\left\{k \leq n: \rho\left(\frac{1}{n_{0}}, \frac{1}{n}\right)<\varepsilon\right\}\right|=1$. This implies that $\left(\frac{1}{n}\right)$ is $a$ weakly left $K$-statistically Cauchy sequence and so it is a left $\rho$-statistically Cauchy sequence in $X$.

For any $x \in X,\left(\frac{1}{n}\right)$ cannot be left $\rho$-statistically convergent to $x$. In fact, firstly take $x=0$. Then, we have that

$$
\left|\left\{k \leq n: \rho\left(0, \frac{1}{k}\right) \geq 1\right\}\right|=\left\{\begin{array}{cl}
\frac{n+1}{2}, & \text { if } n \text { is odd, } \\
\frac{n}{2}, & \text { if } n \text { is even. }
\end{array}\right.
$$

This implies $\lim _{n \rightarrow \infty} \frac{1}{n}\left|\left\{k \leq n: \rho\left(0, \frac{1}{k}\right) \geq 1\right\}\right|=\frac{1}{2}$ and so $\left(x_{n}\right)$ is not left $\rho$-statistically convergent to 0 . Let $x=\frac{1}{N}$ for $a N \in \mathbb{N}$. Then we obtain

$$
n-1 \leq\left|\left\{k \leq n: \rho\left(\frac{1}{N}, \frac{1}{k}\right) \geq \frac{1}{N}\right\}\right| \leq n .
$$

It follows that $\lim _{n \rightarrow \infty} \frac{1}{n}\left|\left\{k \leq n: \rho\left(\frac{1}{N}, \frac{1}{k}\right) \geq \frac{1}{N}\right\}\right|=1$ so $\left(x_{n}\right)$ is not left $\rho$-statistically convergent to $\frac{1}{N}$ for any $N \in \mathbb{N}$ (This is the case of $x \neq 0$ ). Hence, we conclude that $\left(\frac{1}{n}\right)$ is not left $\rho$-statistically convergent. However, it has a left $\rho$-statistically convergent subsequence, say $\left(\frac{1}{2 n}\right)$. Since $\rho\left(0, \frac{1}{2 n}\right)=\frac{1}{2 n}$ for all $n \in \mathbb{N}$, $\left(\frac{1}{2 n}\right)$ is left $\rho$-convergent to 0 and so it is left $\rho$-statistically convergent to 0 .

In particular Example 2.12 implies the following result.

Corollary 2.13. Even if a weakly left $K$-statistically Cauchy sequence or a left $\rho$-statistically Cauchy sequence has a left $\rho$-statistically convergent subsequence, the sequence itself does not need to be left $\rho$-statistically convergent.

The authors of [29] have defined the statistical closure of a set in a cone metric space. According to this definition, a point $x$ belongs to the statistical closure of a set $A$ if there exists a sequence in $A$ which is statistically convergent to $x$. In the same way, we define the statistical closure of $A$ in a quasi-metric space as

$$
\mathrm{Cl}_{s t}(A)=\left\{x \in X: \exists\left(x_{n}\right) \text { in } A \ni x_{n} \stackrel{1-\rho \text { st }}{\longrightarrow} x\right\} .
$$

By $\mathrm{Cl}(A)$ we denote the closure of $A$. But, we observe that $\mathrm{Cl}_{s t}(A)=\mathrm{Cl}(A)$. Indeed, for $x \in \mathrm{Cl}(A)$, there is a sequence $\left(x_{n}\right)$ in $A$ which is left $\rho$-convergent and so left $\rho$-statistically convergent to $x$. This means $x \in \mathrm{Cl}_{s t}(A)$. The reverse implication follows from Corollary 2.10. Hence we conclude that $\mathrm{Cl}_{s t}(A)=\mathrm{Cl}(A)$. Similarly, one can see this in a metric space and a cone metric space.

Now, we give the definition of a left $\rho$-statistical limit point as in [30]. 
Definition 2.14. A point $x$ is said to be a left $\rho$-statistical limit point of a sequence $\left(x_{n}\right)$ in a quasi-metric space $(X, \rho)$ if there is a subset $A=\left\{n_{1}<n_{2}<\ldots<n_{k}<\ldots\right\}$ of $\mathbb{N}$ such that $\lim _{n \rightarrow \infty} \frac{1}{n}|\{k \leq n: n \in A\}|>0$ and the subsequence $\left(x_{n_{k}}\right)$ of $\left(x_{n}\right)$ is left $\rho$-convergent to $x$.

By $\Lambda_{\rho}\left(x_{n}\right)$, we denote the set of all left $\rho$-statistical limit points of the sequence $\left(x_{n}\right)$. By $L_{\rho}\left(x_{n}\right)$, we denote the set of all left $\rho$-limit points of the sequence $\left(x_{n}\right)$. It is clear that if the point $x$ is a left $\rho$-statistical limit point of the sequence $\left(x_{n}\right)$, then it is also a left $\rho$-limit point. The converse is not true in general.

Example 2.15. Let $(X, \rho)$ be the quasi-metric space in Example 2.2. Consider the sequence $\left(x_{n}\right)$ given as

$$
x_{n}=\left\{\begin{array}{c}
1, \quad \text { if } n=m^{2},(m \in \mathbb{N}), \\
0, \quad \text { if } n \neq m^{2} .
\end{array}\right.
$$

By Remark 2.3, we can deduce that $L_{\rho}\left(x_{n}\right)=[0, \infty)$ and $\Lambda_{\rho}\left(x_{n}\right)=[1, \infty)$. The statement $L_{\rho}\left(x_{n}\right)=[0, \infty)$ is clear since $\left(x_{n}\right)$ has no subsequence which is left $\rho$-convergent to $x$ for $x<0$. Now, we prove that a real number $x<1$ cannot be a left $\rho$-statistical limit point. On the contrary, suppose that there exists a subset $\left\{n_{1}<n_{2}<\ldots<n_{k}<\ldots\right\}$ whose asymptotic density is not zero and the subsequence $\left(x_{n_{k}}\right)$ left $\rho$-convergent to $x$. Let $\varepsilon=\frac{1-x}{2}$ and $k_{0} \in \mathbb{N}$. By choosing $n_{k}>k_{0}$ with $n_{k}=m^{2}$ for some $m \in \mathbb{N}$, we obtain $\rho\left(x, x_{n_{k}}\right)=1-x>\varepsilon$ which is a contradiction. So, we conclude that $\Lambda_{\rho}\left(x_{n}\right)=[1, \infty)$.

Corollary 2.16. The strict inclusion $\Lambda_{\rho}\left(x_{n}\right) \subset L_{\rho}\left(x_{n}\right)$ holds.

If a point is a statistical limit point of a sequence (in the sense of the definition in [30]) with respect to the metric $\rho^{s}$, then it is a left $\rho$-statistical limit point of the same sequence in the quasi-metric space.

Example 2.17. Let $(X, \rho)$ be the quasi-metric space in Example 2.2. Consider the sequence $\left(x_{n}\right)=(-n)$. Since $\rho^{s}$ is the usual metric, we have $\Lambda_{\rho^{s}}\left(x_{n}\right)=\emptyset$. Now, we show that $\Lambda_{\rho}\left(x_{n}\right)=\mathbb{R}$. If we choose $x \in[-1, \infty)$, we obtain $\rho\left(x, x_{n}\right)=0$ for all $n \in \mathbb{N}$. Otherwise, if $x \in(-\infty,-1)$, we obtain $\rho\left(x, x_{n}\right)=0$ for all $n \geq|[[x]]|$, where $[[x]]$ denotes the greatest integer less than or equal to $x$. Hence, we conclude that every real number is a left $\rho$-statistical limit point of $\left(x_{n}\right)$.

Corollary 2.18. The strict inclusion $\Lambda_{\rho^{s}}\left(x_{n}\right) \subset \Lambda_{\rho}\left(x_{n}\right)$ holds.

Definition 2.19. A point $x$ is said to be a left $\rho$-statistical cluster point of the sequence $\left(x_{n}\right)$ in a quasi-metric space $(X, \rho)$ if for every $\varepsilon>0$ the asymptotic density of the set $\left\{n: x_{n} \in B_{\rho}(x, \varepsilon)\right\}$ is not zero.

By $\Gamma_{\rho}\left(x_{n}\right)$ we denote the set of all left $\rho$-statistical cluster points of the sequence $\left(x_{n}\right)$.

Theorem 2.20. Let $(X, \rho)$ be a quasi-metric space, $\left(x_{n}\right)$ a sequence in $(X, \rho)$ and $K$ a compact subset of $X$ with respect to the topology $\tau_{\rho}$. If the asymptotic density of the set $\left\{n: x_{n} \in K\right\}$ is not zero, then we have $K \cap \Gamma_{\rho}\left(x_{n}\right) \neq \emptyset$.

Proof. Suppose that $K \cap \Gamma_{\rho}\left(x_{n}\right)=\emptyset$. Then, given any $z \in K$ there exists an $\varepsilon_{z}>0$ such that $\delta\left(\left\{n: x_{n} \in\right.\right.$ $\left.\left.B_{\rho}\left(z, \varepsilon_{z}\right)\right\}\right)=0$. Since $K$ is $\tau_{\rho}$-compact and $\left\{B_{\rho}\left(z, \varepsilon_{z}\right): z \in K, \varepsilon_{z}>0\right\}$ is a left $\rho$-open cover of $K$, we have $K \subset \bigcup_{i=1}^{m} B_{\rho}\left(z_{i}, \varepsilon_{z_{i}}\right)$ for some $z_{i} \in K$ and $\varepsilon_{z_{i}}>0(i=1, \ldots, m)$. Hence, for any $n \in \mathbb{N}$ with $x_{n} \in K$, there exists an $i_{0} \in\{1, \ldots, m\}$ such that $x_{n} \in B_{\rho}\left(z_{i_{0}}, \varepsilon_{z_{i_{0}}}\right)$ which implies $\left\{n: x_{n} \in K\right\} \subset \bigcup_{i=1}^{m}\left\{n: x_{n} \in B_{\rho}\left(z_{i}, \varepsilon_{z_{i}}\right)\right\}$. It follows that $\delta\left(\left\{n: x_{n} \in K\right\}\right) \leq \sum_{i=1}^{m} \delta\left(\left\{n: x_{n} \in B_{\rho}\left(z_{i}, \varepsilon_{z_{i}}\right)\right\}\right)=0$ which is a contradiction.

In [29], statistical completeness is defined as every statistically Cauchy sequence is statistically convergent. Also, it is proved that statistical completeness implies completeness. These notions are equivalent in a metric space, and more generally, in a cone metric space. To see the converse let $X$ be a complete metric space and $\left(x_{n}\right)$ a statistically Cauchy sequence in $X$. By Corollary 3.5 in [31], there exists a Cauchy subsequence $\left(x_{n_{k}}\right)$ such that $\delta\left(\left\{n_{k}: k \in \mathbb{N}\right\}\right)=1$. By completeness, this subsequence is convergent. Statistical convergence of $\left(x_{n}\right)$ 
follows from Lemma $1.1 \mathrm{in}$ [32] (which is also true in a general metric space). We conclude that $X$ is statistically complete.

A quasi-metric space is called sequentially complete [9] if every left $\rho$-Cauchy sequence in $X$ has a left $\rho$-convergent subsequence, that is, it has a left $\rho$-limit point in $X$. By virtue of this notion, we have some results.

Theorem 2.21. A quasi-metric space $(X, \rho)$ is sequentially complete if and only if every left $\rho$-statistically Cauchy sequence in $X$ has a left $\rho$-statistically convergent subsequence.

Proof. Firstly, suppose that $X$ is sequentially complete and let $\left(x_{n}\right)$ be a left $\rho$-statistically Cauchy sequence in $X$. By Corollary 2.10, it has a left $\rho$-Cauchy subsequence. The sequential completeness of $X$ implies that this subsequence has a left $\rho$-convergent and so left $\rho$-statistical convergent subsequence which proves the assertion.

For the converse, let $\left(x_{n}\right)$ be a left $\rho$-Cauchy sequence in $X$. Then, $\left(x_{n}\right)$ is a left $\rho$-statistically Cauchy sequence in $X$ and by hypothesis, it has a left $\rho$-statistically convergent subsequence. It follows from Corollary 2.10 that it has a left $\rho$-convergent subsequence which means that $X$ is sequentially complete.

Theorem 2.22. If every left $\rho$-statistically Cauchy sequence in a quasi-metric space $(X, \rho)$ has a left $\rho$-statistical limit point, then $X$ is sequentially complete.

Proof. Let $\left(x_{n}\right)$ be a left $\rho$-Cauchy sequence in $X$. Then, it is a left $\rho$-statistically Cauchy sequence. By hypothesis, it has a left $\rho$-statistical limit point in $X$ and so it also has a left $\rho$-limit point. Consequently, $X$ is sequentially complete.

In the case of a quasi-metric space, there are several completeness notions (see [4]). One of them is the left $K$ sequential completeness, that is, a quasi-metric space is left $K$-sequentially complete if every left $K$-Cauchy sequence in the space is left $\rho$-convergent. In the same way, the weakly left $K$-sequential completeness is defined as every weakly left $K$-Cauchy sequence in the space is left $\rho$-convergent. Since a left $K$-Cauchy sequence is weakly left $K$-Cauchy, the weakly left $K$-sequential completeness of a quasi-metric space implies the left $K$-sequential completeness. Romaguera [10] remarked that these notions are in fact equivalent. In the following two theorems, we have some results related to these concepts.

Theorem 2.23. A quasi-metric space $(X, \rho)$ is left $K$-sequentially complete if and only if every left $K$-statistically Cauchy sequence in $X$ is left $\rho$-statistically convergent.

Proof. Let $X$ be a left $K$-sequentially complete quasi-metric space and $\left(x_{n}\right)$ be a left $K$-statistically Cauchy sequence in $X$. By Theorem 2.9 there exists a subset $K=\left\{n_{1}<n_{2}<\ldots<n_{k} \ldots\right\}$ of $\mathbb{N}$ such that $\delta(K)=1$ and the subsequence $\left(x_{n_{k}}\right)$ is left $K$-Cauchy. Then, this subsequence is left $\rho$-convergent and again by Theorem 2.9, $\left(x_{n}\right)$ is left $\rho$-statistically convergent.

For the converse, let $\left(x_{n}\right)$ be a left $K$-Cauchy sequence in $X$. Then, $\left(x_{n}\right)$ is a left $K$-statistically Cauchy sequence in $X$ and by hypothesis, it is left $\rho$-statistically convergent. It follows from Corollary 2.10 that it has a left $\rho$-convergent subsequence. The result follows from the fact that if $\left(x_{n}\right)$ is a left $K$-Cauchy sequence which has a left $\rho$-convergent subsequence, then the sequence itself is left $\rho$-convergent (see [2]). Thus, $X$ is left $K$-sequentially complete.

Theorem 2.24. Every left $K$-statistically Cauchy sequence in a quasi-metric space $(X, \rho)$ is left $\rho$-statistically convergent if and only if every weakly left $K$-statistically Cauchy sequence in $X$ is left $\rho$-statistically convergent.

Proof. Firstly, suppose that every left $K$-statistically Cauchy sequence in $X$ is left $\rho$-statistically convergent. Then, from the preceding theorem, we obtain that $X$ is left $K$-sequentially complete. Equivalently, $X$ is weakly left $K$-sequentially complete. Now, let $\left(x_{n}\right)$ be a weakly left $K$-statistically Cauchy sequence in $X$. As explained 
at the first part of the proof of the last theorem, one can say that $\left(x_{n}\right)$ is left $\rho$-statistically convergent. This proves the assertion.

The second part is obvious since a left $K$-statistically Cauchy sequence is also a weakly left $K$-statistically Cauchy sequence.

Lemma 2.25. [5] Let $(X, \rho)$ be a quasi-metric space and $\left(x_{n}\right)$ be a sequence in $X$.

1. If $\left(x_{n}\right)$ is a weakly left $K$-Cauchy sequence, then $\left\{x_{n}: n \in \mathbb{N}\right\}$ is precompact.

2. If $\left(x_{n}\right)$ is left $\rho$-convergent, then $\left\{x_{n}: n \in \mathbb{N}\right\}$ is outside precompact.

Unlike a weakly left $K$-Cauchy or a left $\rho$-convergent sequence, the following example shows that a weakly left $K$-statistical Cauchy or a left $\rho$-statistically convergent sequence may not be outside precompact.

Example 2.26. Let $X=\mathbb{R}$ and $\rho$ be the quasi-metric on $X$ defined by

$$
\rho(x, y)=\left\{\begin{array}{cl}
y-x, & \text { if } x \leq y, \\
1, & \text { if } x>y .
\end{array}\right.
$$

Consider the sequence $\left(x_{k}\right)$ given as

$$
x_{k}=\left\{\begin{array}{cc}
k, & \text { if } k=m^{2},(m \in \mathbb{N}), \\
0, & \text { if } k \neq m^{2} .
\end{array}\right.
$$

Given any $\varepsilon>0$, we have $\left\{k \leq n: \rho\left(0, x_{k}\right)<\varepsilon\right\} \supseteq\left\{k \leq n: k \neq m^{2}, m \in \mathbb{N}\right\}$ and subsequently, $\lim _{n \rightarrow \infty} \frac{1}{n}\left|\left\{k \leq n: \rho\left(0, x_{k}\right)<\varepsilon\right\}\right| \geq \lim _{n \rightarrow \infty} \frac{1}{n}\left|\left\{k \leq n: k \neq m^{2}, m \in \mathbb{N}\right\}\right|=1$. This means that the sequence $\left(x_{k}\right)$ is left $\rho$-statistically convergent and also weakly left K-statistical Cauchy. On the other hand, $\left\{x_{k}: k \in \mathbb{N}\right\}$ is not outside precompact (therefore not precompact) since it cannot be covered by finitely many left $\rho$-balls $B_{\rho}\left(x, \frac{1}{2}\right)=\left[x, x+\frac{1}{2}\right)$ for any $x \in X$.

Also, this sequence is not a left $\rho$-Cauchy sequence so it is neither a weakly left $K$-Cauchy sequence, nor a left $\rho$-convergent sequence. Indeed, given any $x \in \mathbb{R}$, we can find a number $m \in \mathbb{N}$ such that $x+1<m^{2}$ holds. Hence, we obtain $\rho\left(x, x_{k}\right)>1$ for every $k \in \mathbb{N}$ with $k=n^{2}, n \geq m$.

Definition 2.27. A quasi-metric space $(X, \rho)$ is said to be statistically sequentially compact if any sequence in $X$ has a left $\rho$-statistically convergent subsequence.

Theorem 2.28. A quasi-metric space $(X, \rho)$ is statistically sequentially compact if and only if it is sequentially compact.

Proof. Let $X$ be a statistically sequentially compact quasi-metric space and $\left(x_{n}\right)$ be a sequence in $X$. Then, it has a left $\rho$-statistically convergent subsequence and by Corollary 2.10 this subsequence has a left $\rho$ convergent subsequence. This proves sequential compactness of $X$. The converse is obvious.

It is proved in [29] that statistically sequential compactness and compactness are equivalent (the proof is direct). The last theorem can be proved in a similar way for metric or cone metric spaces. Also, since compactness and sequential compactness coincide in a metric or cone metric space, Theorem 3.5 in [29] can be easily seen.

Contrary to the case of a metric space, compactness and sequential compactness are different concepts in a quasi-metric space. It is a well known fact that a (sequentially) compact topological space is countably compact and if a countably compact topological space satisfies first axiom of countability, that is, every point has a countable neighbourhood basis, then it is sequentially compact. Therefore, the sequential compactness and countable compactness are equivalent notions in a quasi-metric space since the set of left $\rho$-open balls centered at $x$ with radius $\frac{1}{n}$ for $n \in \mathbb{N}$ form a countable neighbourhood base at $x$. However, there are examples of a sequentially compact quasi-metric space that is not compact (see [33]). It is shown in [6] that a precompact countably compact quasi-metric space is compact. 
Theorem 2.29. In a precompact quasi-metric space $(X, \rho)$, every sequence has a left $\rho$-statistical Cauchy subsequence.

If $X$ is countable and every sequence in $X$ has a left $\rho$-statistical Cauchy subsequence, then $X$ is precompact.

Proof. Firstly, if $X$ is precompact, then every sequence has a left $\rho$-Cauchy subsequence ([11]) and so it has a left $\rho$-statistical Cauchy subsequence. Conversely, if $X$ is countable and every sequence in $X$ has a left $\rho$ statistical Cauchy subsequence, then by Corollary 2.10 it has a left $\rho$-Cauchy subsequence. It follows that $X$ is precompact.

Theorem 2.30. If $(X, \rho)$ is a precompact quasi-metric space and every left $\rho$-statistically Cauchy sequence in $X$ is left $\rho$-statistically convergent, then $X$ is compact.

Proof. Let $\left(x_{n}\right)$ be a sequence in $X$. Since $X$ is precompact, it has a left $\rho$-statistical Cauchy subsequence. By hypothesis, this subsequence is left $\rho$-statistically convergent and by Corollary 2.10 it has a left $\rho$-convergent subsequence. This implies that $X$ is sequentially compact. Hence, $X$ is compact.

In [34], a hereditarily precompact space is defined as a space where every sequence has a weakly left $K$-Cauchy subsequence. Hence, we prove the following result.

Theorem 2.31. A quasi-metric space $(X, \rho)$ is hereditarily precompact if and only if every sequence in $X$ has a weakly left $K$-statistical Cauchy subsequence.

Proof. If $X$ is hereditarily precompact, then every sequence has a weakly left $K$-Cauchy subsequence and so has a weakly left $K$-statistical Cauchy subsequence.

Conversely, if every sequence in $X$ has a weakly left $K$-statistical Cauchy subsequence, by Corollary 2.10, this subsequence has a weakly left $K$-Cauchy subsequence and so $X$ is hereditarily precompact.

\section{Some results related to summability in asymmetric normed spaces}

In this section, before obtaining some summability type theorems, we give the definition of an asymmetric normed space and related concepts (for the following definitions see [5, 6]). An asymmetric norm on a real vector space $X$ is a mapping $p: X \rightarrow \mathbb{R}$ satisfying the following conditions:

$$
\begin{aligned}
& (\mathrm{AN} 1) p(x)=p(-x)=0 \Leftrightarrow x=0 \\
& (\mathrm{AN} 2) p(\alpha x)=\alpha p(x) \\
& (\mathrm{AN} 3) p(x+y) \leq p(x)+p(y)
\end{aligned}
$$

for all $x, y \in X$ and $\alpha \geq 0$. The ordered pair $(X, p)$ is called an asymmetric normed space. Analogously, the mappings $\bar{p}$ and $p^{s}$ can be defined as $\bar{p}(x)=p(-x)$ and $p^{S}(x)=\max \{p(x), \bar{p}(x)\}$, respectively. The mapping $\bar{p}$ is the conjugate asymmetric norm of $p$ and so $p^{s}$ becomes a norm on $X$. Further, an asymmetric norm $p$ on a real vector space $X$ can be turned into a quasi metric by setting $\rho(x, y)=p(y-x)$ for all $x, y \in X$. A sequence $\left(x_{n}\right)$ in an asymmetric normed space $(X, p)$ is said to be left $p$-convergent to $x \in X$ if $p\left(x_{n}-x\right) \rightarrow 0$ as $n \rightarrow \infty$. A subset $A$ in $(X, p)$ is $p$-bounded if there is a positive constant $M$ such that $p(x) \leq M$ for all $x \in A$.

We define the statistical convergence of a sequence in an asymmetric normed space as follows:

Definition 3.1. A sequence $\left(x_{n}\right)$ in an asymmetric normed space $(X, p)$ is left $p$-statistically convergent to $x \in X$, if the equality $\delta\left(\left\{n: p\left(x_{n}-x\right) \geq \varepsilon\right\}\right)=0$ holds for every $\varepsilon>0$. 
We say that a sequence $\left(x_{n}\right)$ in an asymmetric normed space $(X, p)$ is Cesàro summable to $x \in X$ if the sequence $\left(\frac{x_{1}+\ldots+x_{n}}{n}\right)$ is left $p$-convergent to $x$.

Theorem 3.2. Let $\left(x_{n}\right)$ be a sequence in an asymmetric normed space $(X, p)$. If $\left(x_{n}\right)$ is left $p$-statistically convergent to zero and left $p$-bounded in $X$, then $\left(x_{n}\right)$ is Cesàro summable to zero.

Proof. Suppose that $\left(x_{n}\right)$ is left $p$-statistically convergent to zero and left $p$-bounded in $X$. Then, given any $\varepsilon>0$, we have $\lim _{n \rightarrow \infty} \frac{1}{n}|A(n)|=0$, where $A(n)=\left\{k \leq n: p\left(x_{k}\right) \geq \frac{\varepsilon}{2}\right\}$. Also, we can find a real number $M>0$ such that $p\left(x_{k}\right) \leq M$ for all $k \in \mathbb{N}$. Subsequently, we obtain

$$
p\left(\frac{x_{1}+\ldots+x_{n}}{n}\right) \leq \frac{1}{n}\left(p\left(x_{1}\right)+\ldots+p\left(x_{n}\right)\right) \leq \frac{1}{n}\left(|A(n)| M+(n-|A(n)|) \frac{\varepsilon}{2}\right) \leq \frac{|A(n)|}{n} M+\frac{\varepsilon}{2}<\varepsilon
$$

for all sufficiently large values of $n$. This means that the sequence $\left(\frac{x_{1}+\ldots+x_{n}}{n}\right)$ is left $p$-convergent to zero.

Corollary 3.3. If $\left(x_{n}\right)$ is left $p$-statistically convergent to a point $x \in X$ and left $p$-bounded in $X$, then $\left(x_{n}\right)$ is Cesàro summable to $x$.

We say that a sequence $\left(x_{n}\right)$ in an asymmetric normed space $(X, p)$ is strongly summable to $x \in X$ if $\lim _{n \rightarrow \infty} \frac{1}{n} \sum_{k=1}^{n} p\left(x_{k}-x\right)=0$.

Theorem 3.4. Let $\left(x_{n}\right)$ be a sequence in an asymmetric normed space $(X, p)$. If $\left(x_{n}\right)$ is strongly summable to $x \in X$, then $\left(x_{n}\right)$ is left $p$-statistically convergent to $x$.

Proof. Given any $\varepsilon>0$, we obtain the following inequalities

$$
\frac{\varepsilon}{n}\left|\left\{k \leq n: p\left(x_{k}-x\right) \geq \varepsilon\right\}\right| \leq \frac{1}{n} \sum_{k=1, p\left(x_{k}-x\right) \geq \varepsilon}^{n} p\left(x_{k}-x\right) \leq \frac{1}{n} \sum_{k=1}^{n} p\left(x_{k}-x\right) .
$$

By hypothesis, we have that $\frac{1}{n} \sum_{k=1}^{n} p\left(x_{k}-x\right)<\varepsilon^{2}$ for all sufficiently large values of $n$. It follows that $\lim _{n \rightarrow \infty} \frac{1}{n}\left|\left\{k \leq n: p\left(x_{k}-x\right) \geq \varepsilon\right\}\right|=0$ which implies that $\left(x_{n}\right)$ is left $p$-statistically convergent to $x$.

Theorem 3.5. Let $\left(x_{n}\right)$ be a sequence in an asymmetric normed space $(X, p)$. If $\left(x_{n}\right)$ is left $p$-statistically convergent to $x \in X$ and left $p$-bounded in $X$, then $\left(x_{n}\right)$ is strongly summable to $x$.

Proof. By left $p$-boundedness of $\left(x_{n}\right)$, we can find a real number $M>0$ such that $p\left(x_{n}-x\right) \leq M$ for all $n \in \mathbb{N}$. Also, for every $\varepsilon>0$ there exits $n_{0} \in \mathbb{N}$ such that $\frac{|A(n)|}{n}<\frac{\varepsilon}{2 M}$ for all $n \geq n_{0}$, where $A(n)=\left\{k \leq n: p\left(x_{k}-x\right) \geq \frac{\varepsilon}{2}\right\}$. From the following

$$
\frac{1}{n} \sum_{k=1}^{n} p\left(x_{k}-x\right) \leq \frac{1}{n}\left((n-|A(n)|) \frac{\varepsilon}{2}+|A(n)| M\right)<\frac{\varepsilon}{2}+\frac{\varepsilon}{2 M} M=\varepsilon
$$

we conclude that $\left(x_{n}\right)$ is strongly summable to $x$.

Corollary 3.6. Let $\left(x_{n}\right)$ be a left $p$-bounded sequence in an asymmetric normed space $(X, p)$. Then, $\left(x_{n}\right)$ is left $p$-statistically convergent to $x \in X$ if and only if $\left(x_{n}\right)$ is strongly summable to $x$.

\section{References}

[1] Wilson W. A., On quasi-metric spaces, Amer. J. Math., 1931, 53, 675-684

[2] Reilly I. L., Vamanamurthy M. K., On oriented metric spaces, Math. Slovaca, 1984, 34(3), 299-305

[3] Kelly J. C., Bitopological spaces, J. Proc. London Math. Soc., 1963, 13, 71-89

[4] Reilly I. L., Subrahmanyam P. V., Vamanamurthy M. K., Cauchy sequences in quasi-pseudo-metric spaces, Monatsh. Math., 1982, 93(2), 127-140 
[5] Alegre C., Ferrando I., Garcia-Raffi L. M., Sánchez Pérez E. A., Compactness in asymmetric normed spaces, Topology Appl., 2008, 155(6), 527-539

[6] Cobzaş Ş., Functional Analysis in Asymmetric Normed Spaces, Birkhäuser, Basel, 2013

[7] Collins J., Zimmer J., An asymmetric Arzelà-Ascoli theorem, Topology Appl., 2007, 154(11), 2312-2322

[8] García-Raffi L. M., Compactness and finite dimension in asymmetric normed linear spaces, Topology Appl., 2005, 153, 844853

[9] Künzi H. P., Complete quasi-pseudo-metric spaces, Acta Math. Hungar., 1992, 59(1-2), 121-146

[10] Romaguera S., Left $K$-completeness in quasi-metric spaces, Math. Nachr., 1992, 157, 15-23

[11] Romaguera S., Gutiérrez A., A note on Cauchy sequences in quasi pseudo metric spaces, Glas. Mat. Ser. III, 1986, 21(41)(1), 191-200

[12] Zygmund A., Trigonometric Series, 3rd ed., Cambridge University Press, Cambridge, 2002

[13] Fast H., Sur la convergence statistique, Colloq. Math., 1951, 2, 241-244

[14] Schoenberg I. J., The integrability of certain functions and related summability methods, Amer. Math. Monthly, 1959, 66, 361-375

[15] Fridy J. A., On statistical convergence, Analysis, 1985, 5, 301-313

[16] Fridy J. A., Orhan C., Lacunary statistical summability, J. Math. Anal. Appl., 1993, 173, 497-504

[17] Kirişci M., Karaisa A., Fibonacci statistical convergence and Korovkin type approximation theorems, J. Inequal. Appl., 2017, 2017(1), 229

[18] Küçükaslan M., Değer U., Dovgoshey O., On the statistical convergence of metric-valued sequences, Ukrainian Math. J., 2014, 66(5), 796-805

[19] Di Maio G., Kočinac L. D. R., Statistical convergence in topology, Topology Appl., 2008, 156, 28-45

[20] Mursaleen M., Edely O. H. H., Generalized statistical convergence, Inform. Sci., 2004, 162, 287-294

[21] Dündar E., Ulusu U., Asymptotically I-Cesàro equivalence of sequences of sets, Univers. J. Math. Appl., 2018, 1, 101-105

[22] Kişi Ö, Güler E., J-Cesàro summability of a sequence of order $\alpha$ of random variables in probability, Fund. Jo. Mathe. Appl., 2018, 1, 157-161

[23] Das P., Som S., Ghosal S., Karakaya V., A notion of $\alpha \beta$-statistical convergence of order $\gamma$ in probability, Kragujevac J. Math., 2018, 42(1), 51-67

[24] Edely O. H. H., Mohiuddine S. A., Noman A. K., Korovkin type approximation theorems obtained through generalized statistical convergence, Appl. Math. Lett., 2010, 23, 1382-1387

[25] Belen C., Mohiuddine S. A., Generalized weighted statistical convergence and application, Appl. Math. Comput., 2013, 219, 9821-9826

[26] Kadak U., Mohiuddine S. A., Generalized statistically almost convergence based on the difference operator which includes the $(p, q)$-Gamma function and related approximation theorems, Results Math., 2018, 73(9), 1-31

[27] Toyganözü Z. H., Pehlivan S., Some results on exhaustiveness in asymmetric metric spaces, Filomat, 2015, 29(1), 183-192

[28] Das P., Ghosal S., Pal S. K., Extending asymmetric convergence and Cauchy condition using ideals, Math. Slovaca, 2013, 63(3), 545-562

[29] Li K., Lin S., Ge Y., On statistical convergence in cone metric spaces, Topology Appl., 2015, 196, 641-651

[30] Fridy J. A., Statistical limit points, Proc. Amer. Math. Soc., 1993, 118(4), 1187-1192

[31] Burgin M., Duman O., Statistical convergence and convergence in statistics, arXiv:math/0612179 [math.GM]

[32] Šalát T., On statistically convergent sequences of real numbers, Math. Slovaca, 1980, 30(2), 139-150

[33] Künzi H. P., A note on sequentially compact quasi-pseudo-metric spaces, Monatsh. Math., 1983, 95, 219-220

[34] Künzi H. P., Mršević M., Reilly I. L., Vamanamurthy M. K., Convergence, precompactness and symmetry in quasi-uniform spaces, Math. Japon., 1993, 38, 239-253 